Memorias del VII Encuentro Nacional de Experiencias en la Enseñanza de la Biología y la Educación Ambiental y II Congreso Nacional de Investigación en la Enseñanza de la Biología

\title{
EL USO DE LAS ANALOGÍAS COMO ESTRATEGIA DIDÁCTICA EMPLEADAS POR LOS PROFESORES EN LA ENSEÑANZA DE LA BIOLOGÍA EN LA INSTITUCIÓN EDUCATIVA HÉCTOR JULIO RANGEL QUINTERO, EN EL MUNICIPIO DE FLORESTA- BOYACÁ
}

\section{THE USE OF ANALOGY AS A STRATEGY EMPLOYED BY TEACHING TEACHERS IN THE TEACHING OF BIOLOGY IN COLLEGE HÉCTOR JULIO RANGEL QUINTERO, IN THE MUNICIPALITY OF FLORESTA - BOYACÁ}

\author{
Lizeth Aixa Ariza Bareño ${ }^{1}$ \\ Gloria Escobar ${ }^{2}$
}

\section{Resumen}

Se presenta la propuesta de práctica pedagógica que se desarrolló al interior del Grupo de Investigación "Conocimiento Profesional del Profesor de Ciencias" de la Universidad Pedagógica Nacional. Este proyecto se trabajó en el Municipio de Floresta - Boyacá, en el primer periodo del año 2013 en la Institución Educativa Héctor Julio Rangel Quintero. Para este caso se utilizó el paradigma cualitativointerpretativo;donde se planteó caracterizar las analogías que utilizan los profesores en la enseñanza de la Biología en la institución educativa Héctor Julio Rangel Quintero, y su influencia como estrategia didáctica en el aprendizaje de los estudiantes. Se logró identificar que las profesoras no hacen uso de la explicación en las clases de Biología, ya que abordan la enseñanza de esta disciplina a través de la realización de talleres propuestos en diferentes libros de texto, y la observación de videos relacionados con el tema. También, se logró observar que las profesoras no tienen claro el concepto sobre qué es una analogía, donde se puede intuir que si no hay claridad en este concepto no pueden utilizar las analogías en las clases de Biología como estrategia didáctica, porque no son conscientes de la importancia de la misma.

Palabras Clave: Conocimiento Didáctico del Contenido, Analogía, Enseñanza de la Biología, contexto.

\section{Abstract}

Wepresenttheproposedpedagogicalpracticethatdevelopedwithintheresearchgroup "Teacher Professional Knowledge Science" NationalPedagogicalUniversity. Thisprojectworked in themunicipalityof Floresta - Boyacá, in thefirstquarterof 2013 in theEducationallnstitution Hector Julio Rangel Quintero. For this case weused

\footnotetext{
${ }^{1}$ Universidad Pedagógica Nacional. Estudiante de Licenciatura en Biología. lisa19111@hotmail.com

${ }^{2}$ Universidad Pedagógica Nacional. Profesora del Departamento de Biología . araneida542@yahoo.com.mx
} 
Memorias del VII Encuentro Nacional de Experiencias en la Enseñanza de la Biología y la Educación Ambiental y II Congreso Nacional de Investigación en la Enseñanza de la Biología

the qualitative - interpretativeparadigm, whichraisedcharacterizetheanalogiesused by teachers in theteachingofbiology in school Hector Julio Rangel Quintero, and its influence as a teaching strategy on studen tlearning. We identified that teachers do notmake use ofexplanation in biology classes as they tackletheteachingofthis discipline through the proposed workshops in different books, and watching vídeo son the topic. Also, note that the teachers didnot have a clear concep tofwhatan analogy, where one cansenset hatifthereis no clarity on this concep tcannot use analogies in biology classes as a teachingstrategy, becausethey are umawareoftheimportanceof it.

Key Words: Pedagogical content knowledge, Analogy, Biology teaching, context.

\section{Introducción}

En la formación de profesores de Biología se requiere continuamente la reflexión, consolidación y consenso de aquellos contenidos con los cuales los profesores construyen sus teorías y estrategias de enseñanza, en un contexto escolar determinado, de modo que puedan dar respuesta a las necesidades actuales de una educación científica de calidad para todos y todas. Esta idea básica permite el planteamiento de trabajos de investigación en diferentes áreas como lo es el Conocimiento Profesional del Profesor, y particularmente en el marco de estudio del Conocimiento Didáctico del Contenido, el cual hace referencia a las estrategias metodológicas y didácticas que usa el profesor en sus clases diarias. El interés de la presente investigación se centra en una de las estrategias de enseñanza más utilizadas en la enseñanza de la biología: las analogías.

El trabajo que se presenta a continuación aborda la problemática sobre la caracterización de las analogías que usan los profesores en la enseñanza de la Biología, particularmente en el Municipio de Floresta - Boyacá, y su influencia como estrategia didáctica en el aprendizaje de los estudiantes.

Floresta es un municipio ubicado en el macizo de la cordillera oriental, en el departamento de Boyacá-Colombia. Posee varios afloramientos paleontológicos, de la era Paleozoica del periodo Devónico aproximadamente 350 millones de años. La fauna Florestana está compuesta Briozoos, Braquiópodos, crinoideos, corales, ostrácodos, trilobites y pelecípodos del devónico medio (Cediel, 1969. Citado en Suarez, 2010).

A nivel educativo este municipio cuenta con una Institución Educativa oficial llamada Héctor Julio Rangel Quintero, dondeen el caso urbano se puede encontrar la educación Básica primaria y Básica secundaria, con especialización técnica en dos modalidades, una para el área de Electricidad y la otra modalidad en el área de Comercio, mientras que a nivel rural se encuentra a manera de Escuela Nueva, con los grados correspondientes de la básica primaria. Para el 
Memorias del VII Encuentro Nacional de Experiencias en la Enseñanza de la Biología y la Educación Ambiental y II Congreso Nacional de Investigación en la Enseñanza de la Biología

área de Ciencias Naturales (Biología y Química) la institución del casco urbano cuenta con dos profesoras en el nivel secundario, La profesora número uno, tiene una formación en Licenciatura en Química y Biología, es egresada de la Universidad Pedagógica y Tecnológica de Colombia (UPTC). Ejerciendo la profesión Docente desde hace 20 años, pero en esta institución desde hace 10 años aproximadamente. La profesora número dos tiene una formación en Licenciatura en Biología y Química de la Universidad Pedagógica y Tecnológica de Colombia (UPTC), trabajando como docente vinculada con la gobernación de Boyacá desde hace 32 años.

Teniendo en cuenta el contexto (Floresta - Boyacá) en el que se desenvuelven tanto los estudiantes como los profesores, los contenidos disciplinares debieran estar íntimamente relacionados con los conocimientos cotidianos, llevando a la necesidad de que las analogías que el profesor pretenda utilizar, emplear, introducir y evaluar, dependen de esta relación. En este sentido la efectividad de utilizar una estrategia didáctica como las analogías, dependerá entonces de la relación de estas con los sujetos en su contexto.

\section{Metodología}

Este proyecto se trabajó en el Municipio de Floresta - Boyacá, en el primer periodo del año 2013 en la Institución Educativa Héctor Julio Rangel Quintero. Para este caso se utilizo el paradigma cualitativo-interpretativo que está estrechamente relacionado con la investigación o perspectiva cualitativa (Cerda, 2001); metodológicamente este paradigma está caracterizado por el énfasis que hace en las técnicas de descripción, clasificación y explicación. Es allí donde toma relevancia este paradigma ya que a través de las analogías, se emplean varias estrategias de enseñanza, que permiten que el estudiante relacione dos o más conceptos, y encuentre semejanzas entre éstos, generando razonamientos y conductas basándose en la existencia de las semejanzas entre unos, creando un nuevo campo de conocimiento. Logrando así que los estudiantes comprendan los contenidos de la enseñanza de la Biología, en nuestro caso. Como forma de razonamiento, el Método Inductivo hace posible el pasode los hechos singulares a los principios generales (Cerda, 2001). De esta forma la analogía permite una forma inductiva de argumentar, en que si dos o más entidades son semejantes en uno o más aspectos, entonces es probable que existan entre ellos más semejanzas. Por consiguiente, en el proceso mental el pensamiento se mueve por inducción del conocimiento de los fenómenos parciales al conocimiento del todo. (Cerda, 2001).

Para esto se utilizaron instrumentos de recolección de información que nos permitieron responder a los objetivos de la práctica, como la 'observación de clases', y 'entrevistas estructuradas', las cuales estuvieron dirigidas a las profesoras mencionadas anteriormente, para identificar las analogías que estas 
Memorias del VII Encuentro Nacional de Experiencias en la Enseñanza de la Biología y la Educación Ambiental y II Congreso Nacional de Investigación en la Enseñanza de la Biología

utilizan en la enseñanza de la Biología y su influencia como estrategia didáctica en el aprendizaje de los estudiantes, e intentar dar respuesta a esta problemática. Para el análisis de la información que se obtuvo de las entrevistas se realizo por medio de análisis de contenido. Los datos obtenidos nos permitieron construir tablas de resultados para realizar una interpretación cualitativa y así caracterizar las analogías y evidenciar el uso que hacen de ellas los profesores en la enseñanza de la biología, y su relación con el contexto, tanto de los profesores como el de los estudiantes.

El diseño metodológico se realizó en varias etapas que son enunciadas y resumidas a continuación:

\begin{tabular}{|l|l|l|l|l|}
\hline $\begin{array}{l}\text { Prácti } \\
\text { ca } \\
\text { Integr } \\
\text { al }\end{array}$ & $\begin{array}{l}\text { Etapa 1 } \\
\text { Inicio de la } \\
\text { práctica } \\
\text { profesional }\end{array}$ & $\begin{array}{l}\text { Etapa 2 los } \\
\text { Durante } \\
\text { procesos de } \\
\text { prácticaprofesional }\end{array}$ & $\begin{array}{l}\text { Etapa 3 terminar la práctica } \\
\text { profesional }\end{array}$ & $\begin{array}{l}\text { Etapa 4 } \\
\text { Finalización } \\
\text { de la } \\
\text { práctica } \\
\text { profesional }\end{array}$ \\
\hline & $\begin{array}{l}\text { Proceso de } \\
\text { Contextuali } \\
\text { zación. }\end{array}$ & $\begin{array}{l}\text { Recolección de } \\
\text { Información } \\
\text { mediante: } \\
\text { *Observaciones de } \\
\text { clases. } \\
\text { *Aplicación de de } \\
\text { entrevistas } \\
\text { estructuradas para } \\
\text { profesores }\end{array}$ & $\begin{array}{l}\text { Análisis de la información } \\
{ }^{*} \text { Construcción de tablas } \\
\text { de resultados para una } \\
\text { interpretación cualitativa. } \\
\text { *Sistematización } \\
\text { información. }\end{array}$ & $\begin{array}{l}\text { Construcción } \\
\text { final del } \\
\text { documento } \\
\text { de práctica } \\
\text { pedagógica. }\end{array}$ \\
\end{tabular}

\section{Resultados Y Discusión}

De acuerdo con el proceso de investigación se diseñó e implemento una entrevista estructurada dirigida a dos profesoras de la institución educativa Héctor Julio Rangel Quintero en el Área de ciencias naturales. Se realizaron preguntas relacionadas con los componentes del Conocimiento Profesional del Profesor de Ciencias que deben de tener en cuenta los profesores a la hora de realizar los procesos de enseñanza - aprendizaje en nuestro caso referidos a la Biología.

Teniendo en cuenta que el Conocimiento Profesional del Profesor de Ciencias está relacionado con al menos cuatro componentes desde los cuales se desarrollan las actividades de enseñanza. Se realizará un análisis general desde las preguntas que responden a estos componentes. Donde el primero está referido al conocimiento disciplinar; el segundo se refiere a los conocimientos pedagógicos; el tercer hace énfasis en los aspectos didácticos de la práctica de enseñanza; y el último al conocimiento del contexto (Valbuena, 2007). 
Memorias del VII Encuentro Nacional de Experiencias en la Enseñanza de la Biología y la Educación Ambiental y II Congreso Nacional de Investigación en la Enseñanza de la Biología

Es importante tener en cuenta que en la mayoría de las preguntas que se realizaron a las dos profesoras, no respondieron adecuadamente a la mayoría de las preguntas formuladas, para lo cual en muchas ocasiones fue difícil realizar un análisis desde lo que ellas respondieron. Por ende muchos de los análisis planteados en el apartado del Conocimiento Profesional del Profesor de Ciencias se realizan no solo desde las respuestas dadas por ellas sino que se contrastaba también desde lo observado.

De acuerdo a lo anterior, las preguntas número 5, 7, y 13 estaban planteadas de tal modo que respondieran y se relacionan con el Conocimiento Disciplinar. Donde se puede encontrar que este conocimiento es necesario tanto a nivel estructural como a nivel de su naturaleza, puesto que nos permite saber acerca de la disciplina, en nuestro caso la Biología y las relaciones que existen entre los conceptos que se enseñan y las aplicaciones que puedan tener los contenidos a la cotidianidad de los alumnos. Al igual, también permite identificar los principios fundamentales de la disciplina, y en esa medida seleccionar, secuenciar y transformar los contenidos de enseñanza prioritarios, y por ultimo evaluar y seleccionar textos escolares, teniendo en cuenta la organización de los contenidos. Gess-Newsome, 1999 (citados en Valbuena, 2007). Por ende se puede analizar que aunque las profesoras tengan conocimiento acerca de la disciplina, en muchas ocasiones no lo enseñan a partir de una explicación a sus estudiantes sino que simplemente realizan la aplicación de talleres para abordar los temas. Al igual que este conocimiento les permite seleccionar los libros de texto, en este caso son los de editorial Santillana, en mayor medida, ya que en ellos hay gran cantidad de talleres que pueden aplicar a sus estudiantes, pero no observan que en algunos de esos libros encontramos errores conceptuales que si no se detectan a tiempo pueden generar problemas en la enseñanza - aprendizaje de los contenidos. Por último es importante mencionar que las profesoras tienen creencias religiosas fuertemente arraigadas, las cuales se convierten en un obstáculo para enseñar evolución, teniendo en cuenta que esta temática es de gran importancia en la enseñanza de la Biología, debe ser incluida dentro de sus clases de Biología. Así cuando expliquen este tipo de temáticas deben tener claro que lo que se busca es mostrar otras teorías científicas que se han propuesto alrededor de la vida, sin cambiar las creencias religiosas de ellas mismas y mucho menos de los estudiantes.

Por otra parte, las preguntas número 3 y 4 estuvieron relacionadas y planteadas desde el Conocimiento Pedagógico, el cual está relacionado con las concepciones sobre la enseñanza-aprendizaje, la organización y administración en el aula de clase, los principios generales de la instrucción, los sistemas de evaluación, etc. (Grossman, 1990, citado en Valbuena, 2007). Por ende, cuando se indaga en las profesoras de cuándo y porqué utiliza las estrategias didácticas, ellas no responden adecuadamente a las preguntas. Entonces desde lo observado, se puede analizar que ellas muchas veces no organizan sus clases 
Memorias del VII Encuentro Nacional de Experiencias en la Enseñanza de la Biología y la Educación Ambiental y II Congreso Nacional de Investigación en la Enseñanza de la Biología

adecuadamente porque, como lo expresan, "enseñar Biología es más fácil" y por medio de lecturas y aplicación de talleres se puede aprender sobre esta disciplina. También porque en muchas ocasiones no utilizan las estrategias que dicen para desarrollar su práctica pedagógica.

En cuanto a las preguntas número 1, 2, 8, 9, 11, 12, estas estaban relacionadas con Conocimiento Didáctico del contenido, uno de los aspectos más relevantes dentro del CPPC y en el cual se centraba la mayor parte de nuestra atención ya que desde allí los profesores utilizan diferentes estrategias metodológicas y didácticas para enseñar la disciplina y resolver problemas que están presentes en el contexto escolar.De acuerdo a esto se encuentra una falencia por parte de las profesoras ya que no tienen claro qué es una estrategia didáctica y suelen confundirlas con herramientas pedagógicas, haciendo en mucha ocasiones referencia a éstas como estrategias didácticas.

También se puede evidenciar que aunque ellas mencionan una larga lista acerca de las estrategias que utilizan en su práctica cotidiana, en mayor medida utilizan la resolución de talleres propuestos por los libros de texto, y la observación de videos de la editorial Arisma S.A., haciendo que los estudiantes generen un rechazo hacia el aprendizaje de la materia o que en muchas ocasiones generen un proceso memorístico de la información contenida en los libros de texto.

Por otra parte, es importante resaltar que dentro de las estrategias particulares en la enseñanza de la biología es frecuente el uso de 'analogías' como estrategia didáctica para acercar los contenidos disciplinares al conocimiento propio del estudiante. Por ende a la hora de realizar el proceso de indagación acerca del tema de interés de esta investigación (las analogías) se esperaba que las profesoras en muchos casos usaran esta estrategia didáctica para establecer relaciones de semejanza entre dos o más cosas (Gallego, 2004) y a la vez para resolver problemas que se encuentran en el contexto escolar, para así poder realizar una caracterización de esas analogías y analizar su influencia como estrategia didáctica. Donde se encontró todo lo contrario a esto, ya que ellas en sus clases de biología no hacen uso de la explicación en gran parte, por ende no utilizan las analogías. Aunque cabe aclarar que ellas utilizan las analogías en las clases de química pero que, en muchas ocasiones no están conscientes de que las utilizan y que en este caso las analogías hacen parte de las estrategias didácticas.

Y por último encontramos las preguntas número 6 y 10 las cuales están relacionadas con el Conocimiento del Contexto donde Barnett y Hodson (2001) (citados en Valbuena, 2007) resaltan que en la profesión los profesores no se limitan a aplicar los currículos generalizados que han realizado los expertos, los cuales suelen estar distantes de la realidad escolar. Por el contrario, responden a estos currículos atendiendo a sus creencias personales, sus valores y 
Memorias del VII Encuentro Nacional de Experiencias en la Enseñanza de la Biología y la Educación Ambiental y II Congreso Nacional de Investigación en la Enseñanza de la Biología

experiencias. Entonces debemos tener en cuenta que aunque las profesoras pertenecen a la institución no siempre enseñan los contenidos relacionándolos con el contexto de los estudiantes que en este caso es Floresta Boyacá. Aun sabiendo que Floresta está relacionado con una temática particular de gran importancia en la Biología (la Evolución), y se olvidan de que Floresta tiene una gran riqueza alrededor de fósiles correspondientes al periodo Devónico del Paleozoico (Alcaldía Municipal de Floresta, 2012) la cual ha sido aprovechada por personas ajenas al municipio.

\section{Conclusiones}

Se puede identificar que las profesoras no tiene muy claro el concepto sobre qué es una analogía, donde se puede intuir a la vez que si no hay claridad en este concepto no pueden utilizar las analogías en las clases de Biología como estrategia didáctica, porque no son conscientes de la importancia de la misma.

Es importante que las profesoras de Ciencias Naturales de la Institución Héctor Julio Rangel Quintero reflexionen acerca de las prácticas de enseñanza que están ejerciendo en el área de Biología, y muestre un poco más de interés por esta disciplina como lo hacen por la Química, ya que si tenemos en cuenta la Biología también es una disciplina importante que debemos aprender; y que si aplicamos buenas metodologías y estrategias de enseñanza seguramente podemos despertar más interés por parte de los estudiantes para el aprendizaje de la misma, y por ende podríamos realizar grandes aportes al municipio para que no se desaproveche la riqueza que posee el contexto Florestano.

Esta práctica pedagógica me generó constantes reflexiones acerca de mi que hacer como maestro ya que a medida que pasaba el tiempo, los profesores de la institución hacían comentarios acerca de lo agotador que es la profesión docente y la manera como al transcurrir de los años su profesión se vuelve en un proceso monótono que se realiza solo por el recurso económico y con las ganas de obtener una pensión para poder descansar de tanto estrés que ésta genera. Pero a pesar de esto, en muchas ocasiones no compartía sus pensamientos ya que dentro de mis planes y metas profesionales la docencia es algo que me apasiona, donde espero realizar este proceso con esmero, dedicación y mucha profesionalidad. Para poder transformar este pensamiento.

Hay un desconocimiento y desaprovechamiento del contexto por parte de las profesoras, puesto que como ellas expresan, en muchas ocasiones no enseñan temáticas de la Biología, como la evolución, puesto que no creen en eso por sus creencias religiosas, y por ende no ven la importancia y riqueza que posee Floresta y cómo si empleamos las analogías puede ser excelente estrategia para la enseñanza de esta temática y de muchas más. 
Bio-grafía Escritos sobre la Biología y su Enseñanza.

Edición Extra-Ordinaria. ISSN 2027-1034 P.p 556 - 563

Memorias del VII Encuentro Nacional de Experiencias en la Enseñanza de la Biología y la Educación Ambiental y II Congreso Nacional de Investigación en la Enseñanza de la Biología

Por medio de la práctica confirmé y ratifiqué mi deseo por la profesión docente, para ayudar a diferentes personas en la construcción del conocimiento, en este caso por la biología y generar en mis estudiantes un deseo hacia su conocimiento

\section{Bibliografía}

ALCALDÍA MUNICIPAL DE FLORESTA - BOYACÁ. (2012). Información general del municipio de floresta- Boyacá, Colombia. www.floresta-boyaca.gov.co

BROMME, R. (1988). Conocimientos profesionales de los profesores. Enseñanza de las Ciencias, 6 (1). Pp.19-29.

CERDA, H. (2001). Los elementos de la investigación: como reconocerlos, diseñarlos y construirlos. Editorial el Búho. Bogotá. Colombia.

GALLEGO, R. (2004). Un concepto epistemológico de modelo para la didáctica de las ciencias experimentales. Enseñanza de las Ciencias. 3 ( 3). Pp. 301-319.

OLIVA, J. y ADURIZ BRAVO, A. (2005). Simposio "Las analogías en la enseñanza de las ciencias". Enseñanza de las Ciencias. Pp. 1-2.

SUAREZ, T. (2010). Plan de manejo museográfico para el municipio de Floresta, Boyacá. Tesis de Pregrado. Universidad Nacional de Colombia. Bogota. Pp. 1-84.

VALBUENA, E. (2007). El Conocimiento Didáctico del Contenido Biológico. Estudio de las concepciones disciplinares y didácticas de futuros docentes de la Universidad Pedagógica Nacional (Colombia). Tesis doctoral. Universidad Complutense de Madrid. España. 